TITLE:

\title{
Expression dynamics and functions of Hes factors in development and diseases.
}

$\operatorname{AUTHOR}(\mathrm{S})$ :

Kobayashi, Taeko; Kageyama, Ryoichiro

\section{CITATION:}

Kobayashi, Taeko ...[et al]. Expression dynamics and functions of Hes factors in

development and diseases.. Current topics in developmental biology 2014, 110: 263-283

\section{ISSUE DATE:}

2014

URL:

http://hdl.handle.net/2433/191118

\section{RIGHT:}

(c) 2014 Elsevier Inc.; This is not the published version. Please cite only the published version.; この論文は出版社版でありません。引用の際に は出版社版をご確認ご利用ください。 


\section{Expression dynamics and functions of Hes factors in development and diseases}

Taeko Kobayashi ${ }^{1,2} *$ and Ryoichiro Kageyama ${ }^{1,2,3} *$

${ }^{1}$ Institute for Virus Research, Kyoto University, Shogoin-Kawahara, Sakyo-ku, Kyoto 606-8507, Japan.

2 Japan Science and Technology Agency, Core Research for Evolutional Science and Technology (CREST), 4-1-8 Honcho, Kawaguchi, Saitama, 332-0012, Japan.

${ }^{3}$ World Premier International Research Initiative-Institute for Integrated Cell-Material Sciences (WPI-iCeMS), Kyoto University, Kyoto 606-8501, Japan.

*Correspondence to: Ryoichiro Kageyama, Institute for Virus Research, Kyoto University, Shogoin-Kawahara, Sakyo-ku, Kyoto 606-8507, Japan. E-mail: rkageyam@virus.kyoto-u.ac.jp ; Taeko Kobayashi, Institute for Virus Research, Kyoto University, Shogoin-Kawahara, Sakyo-ku, Kyoto 606-8507, Japan. E-mail: tkobayas@virus.kyoto-u.ac.jp 


\begin{abstract}
Hes genes, encoding basic helix-loop-helix (HLH) transcriptional repressors, are mammalian homologues of Drosophila hairy and Enhancer of split genes, both of which are required for normal neurogenesis in Drosophila. There are seven members in the human Hes family, Hesl to Hes 7, which are expressed in many tissues and play various roles mainly in development. All Hes proteins have three conserved domains: basic HLH (bHLH), Orange, and WRPW domains. The basic region binds to target DNA sequences while the HLH region forms homo- and hetero-dimers with other bHLH proteins, the Orange domain is responsible for the selection of partners during heterodimer formation, and the WRPW domain recruits co-repressors. Hes1, Hes5, and Hes 7 are known as downstream effectors of canonical Notch signaling, which regulates cell differentiation via cell-cell interaction. Hes factors regulate many events in development by repressing the expression of target genes, many of which encode transcriptional activators that promote cell differentiation. For example, Hes $1, H e s 3$, and Hes 5 are highly expressed by neural stem cells, and inactivation of these genes results in insufficient maintenance of stem cell proliferation and prematurely promotes neuronal differentiation. Recently, it was shown that the expression dynamics of Hes1 plays crucial roles in proper developmental timings and fate determination steps of embryonic stem cells and neural progenitor cells. Here we discuss some key features of Hes factors in development and diseases.
\end{abstract}

Key words: Hes, stem cells, differentiation, Hes1 oscillation, embryonic stem cells, neural stem cells 


\section{Introduction}

Development is a highly organized process that depends on the timely proliferation of stem cells and their differentiation into multiple cell types. The balance between cell proliferation and cell fate determination is critical to forming organs with the right shape and size and the right cell composition. Hes factors (hairy and Enhancer of split homologs) play important roles in the development of many organs to expand progenitor cell pools and regulate cell fate decisions. Some Hes factors, Hes1, Hes5, and Hes7, are dynamically expressed by auto-negative feedback loops, and also function as effectors of the Notch signaling pathway, which coordinates cell proliferation and differentiation via cell-cell interaction. Recent studies have shown that the expression dynamics of Hes 1 contribute to its multiple functions. In this review, we describe the features of Hes factors, their functions in development and diseases, and the significance of Hes 1 oscillation, including its expression dynamics in embryonic stem cells and neural progenitor/stem cells.

\section{Hes family members and protein structures}

The protein products of the Hes gene family comprise seven members in human, Hes 1 to Hes7. Hes1, Hes2, and Hes4 are more similar to Drosophila hairy in their amino acid sequences than are the other members (Fig. 1A). Hes4 is the most similar to Hes1 and is not present in mouse or rat. Hes 3 encodes two isoforms due to alternative splicing: Hes3 variant 1 lacks the N-terminal half of the basic region for DNA binding, while Hes 3 variant 2 has a complete basic region (Hirata, et al., 2000; Katoh \& Katoh, 2007). Hes factors have three conserved domains: the basic helix-loop-helix (bHLH) domain, the Orange domain, and the WRPW domain, harboring a WRPW motif (Trp-Arg-Pro-Trp) (Fig.1B) (Kageyama, et al., 2007). There are also other Hes-related bHLH factors such as Hey1, Hey2, HeyL, and HELT (Hes/Hey-like), that do not have the WRPW motif.

The bHLH domain is essential for DNA binding and homo- and heterodimer formation. The N-terminal basic region plays an important role in the specific binding to target DNA sequences, that is, the $\mathrm{N}$ box (CACNAG) and the class $\mathrm{C}$ site (CACG(C/A)G) (Sasai, et al., 1992). It has been suggested that a proline residue in the middle of the basic region (Fig. 1B), which is conserved in all Hes factors as well as Drosophila hairy and Enhancer of split $(\mathrm{E}(\mathrm{spl}))$ proteins, may be involved in the target sequence specificity, providing a higher affinity for the $\mathrm{N}$ box and the class $\mathrm{C}$ site than 
for the E box (CANNTG), a consensus binding sequence for activator-type bHLH factors. The HLH domain is characterized by two alpha-helix motifs linked by a flexible loop structure, and interacts with other HLH motifs of binding partner proteins to form homo- or hetero-dimers. Hes factors can form homo-dimers and hetero-dimers with Hes-related bHLH repressors, such as Hey factors, as well as with other bHLH factors, such as Mash1, E47, and Id. The Orange domain has two amphipathic helixes that are involved in the selection of partners to form hetero-dimers. The WRPW domain, located at the $\mathrm{C}$-terminal end, is essential for the repressor activity via recruitment of the co-repressor Transducin-like E(spl) (TLE1-4)/ Groucho-related gene (Grg), a homologue of Drosophila Groucho. The Hey family factors contain a YRPW (Tyr-Arg-Pro-Trp) motif instead of the WRPW motif, which contributes to the weaker binding of Hey factors to TLE than the Hes factors (Iso et al., 2001; Fischer \& Gessler, 2007).

\section{Hes general functions}

Hes factors repress transcription by two different mechanisms, active repression and passive repression (Fig. 1C) (Kageyama et al., 2007). During active repression, Hes factors form homodimers or heterodimers with other Hes/Hey factors and bind to the $\mathrm{N}$-box or the class $\mathrm{C}$ site sequences in the promoter region of target genes. In the case of the heterodimers between Hes1 and Hey1 or Hey2, binding to the class $\mathrm{C}$ site exhibits a higher affinity than Hes1 or Hey1 homodimers, and such heterodimers repress transcription more efficiently than the homodimers. The C-terminal WRPW domain in Hes factors interacts with TLE/Grg co-repressors (Buscarlet et al., 2008), which recruit histone deacetylase and repress the target gene expression by inducing changes in the chromatin structures. Hes 1 can also repress target genes in a TLE-independent manner, by recruiting Sirt1, a homologue of silent information regulator 1 and a class III histone deacetylase, via its bHLH domain, and thereby repressing target genes (Takata \& Ishikawa, 2003). Although Hes family genes encode transcriptional repressors, there is one exceptional gene, Hes6. The Hes6 protein has no DNA binding ability and inhibits Hes1 function by forming heterodimers with it.

During passive repression, Hes factors form heterodimers with bHLH-type activators, such as Mash1 and E47, and inhibit their binding to the E box in the promoter region of target genes (Akazawa, et al., 1992). Thus, Hes factors function as dominant negative regulators to these bHLH activators. Id proteins, which lack the basic DNA binding domain, also negatively regulate bHLH activators by inhibiting their 
DNA binding through heterodimerization. Hes factors can tightly repress the expression of target genes via these two types of repression. For example, Hes 1 represses Mash1, a mouse homologue of Drosophila achate-scute genes that encode transcriptional activators and promote neural differentiation, both via active repression by directly binding to the class C-site on the Mash1 promoter and via passive repression by capturing E-protein activators such as E47 (Fig. 1C).

\section{Roles of Hes factors in various tissues}

Hes gene expression is regulated by the Notch-dependent signaling pathway (Fig. 2). Notch signaling is activated when ligands such as Delta or Jagged expressed on the surface of neighboring cells bind to Notch receptors. Upon activation of Notch signaling, the Notch intracellular domain (ICD) is released from the cell surface and is translocated into the nucleus, where it forms a complex with the DNA-binding protein $\mathrm{RBPj} / \mathrm{CSL}$. The RBPj-ICD complex recruits additional transcriptional co-activators and induces the expression of downstream genes, including Hes and Hey. This pathway is often called the canonical pathway of Notch signaling. Mouse Hes1, Hes5, and Hes7, which are all activated by Notch signaling, have RBPj binding sites on their promoters (Bessho, et al., 2001a; Katoh \& Katoh, 2007; Ohtsuka et al., 1999). On the other hand, other signaling pathways are also reported to induce Hes expression. For example, Hes1 expression is induced by many signaling pathways, including bone morphogenetie protein (BMP), fibroblast growth factor (FGF), leukemia inhibitory factor (LIF), NF- $\kappa B$, Sonic hedgehog (Shh), and Wnt signaling pathways (Kageyama, et al., 2006; Nakayama, et al., 2008). Hes 1 regulates the proliferation of neural stem/progenitor (NS) cells in the central (see below) and peripheral nervous systems under the control of these pathways. Hes7, a segmentation clock gene involved in somitogenesis, is regulated by the Notch and FGF pathways during somite segmentation (Niwa et al., 2007). Thus, many pathways regulate Hes expression in a tissue-specific manner.

Hes genes are involved in regulation of many different cell types, including hematopoietic stem cells in embryos (Kageyama et al., 2007), digestive organs, lung, cochlea, skeletal tissues, testis and vascular endothelial cells, as discussed below. In digestive organs, Hes 1 and Hes5 are expressed by proliferating progenitor/stem cells, and play an important role in the maintenance of these cells by preventing their differentiation. Hes 1 is detected in the developing pancreas, where it represses the expression of the bHLH-type activators Neurogenin3 (Ngn3) and pancreas transcription factor 1 (Ptfla), which enhance the differentiation of endocrine and exocrine cells, 
respectively. Hes1 maintains progenitors by inhibiting the expression of these bHLH activators. Genetic inactivation of Hes1 up-regulates the expression of these bHLH activators, resulting in, for example, severe pancreatic hypoplasia and the formation of an ectopic pancreas (Fukuda et al., 2006; Jensen et al., 2000; Sumazaki et al., 2004). Hes 1 is also expressed in the intestinal crypt, where stem cells and proliferating progenitors are located, and represses the expression of bHLH-type activator genes, Math1 and Ngn3, whose translational products promote the differentiation of secretory cells. Math1 regulates the development of all types of secretory cells, while Ngn3 regulates enteroendocrine cell development. Hes 1 knockout (KO) mice showed an overproduction of secretory cells (Jensen et al., 2000). Importantly, Hes 1 inactivation markedly reduces tumor cell proliferation and induces their differentiation into intestinal epithelial cells without affecting the homeostasis of normal intestinal crypts in adenomatous polyposus coli (Apc) mutation-induced intestinal tumors, suggesting that Hes1 is a promising target for intestinal cancer therapy (Ueo et al., 2012).

In sensory organs, it has been reported that Hes and Hes-related genes are expressed in the developing inner ear and the developing retina. Hes1, Hes5, and Hey1 are expressed in the developing cochlea of the inner ear, and they cooperatively inhibit the formation of hair cells, which are sensory neurons. Inactivation of these genes leads to disorganized cell alignment and polarity and to hearing loss due to hair cell overproduction (Tateya, et al., 2011). In the developing retina, Hes 1 and Hes5 function downstream of both Shh and Notch signaling, inhibit the activity of proneural bHLH genes such as Mash1 and Math3, and maintain progenitor cell populations (Wall, et al., 2009; Ohtsuka, et al., 2001). Hes6 is exceptionally expressed in the differentiated neural retina and functions as an inhibitor of Hes1, thereby promoting neuronal differentiation (Bae, et al., 2000).

In hematopoietic stem cells (HSC), both Hes1 and Hes5 are induced by Notch signaling and regulate several blood lineages, including T cells, B cells, and stem cells. Forced expression of Hes 1 results in increased numbers of quiescent HSCs, and Hes 1-deficient HSCs are unable to differentiate into T cells, suggesting that Hes1 is an important regulator for HSC functions (Tomita et al., 1999). It has been shown that, although Hes 1-deficient mice do not show clear abnormalities in their hematopoietic system, the depletion of both Hes 1 and Hes 5 induces overproduction of nonfunctional HSCs, in which Hes target genes such as Gata2 are abnormally up-regulated (Guiu et al., 2013). Hes 1 is also reported to be an important regulator of the development of $\mathrm{T}$ cell acute lymphoblastic leukemia (Espinosa et al., 2010; Wendorff et al., 2010). 
Hes 7 is a segmentation clock gene involved in somitogenesis of embryos (Bessho, et al., 2001a; Bessho, et al., 2001b). Somites are metameric structures periodically segmented from the anterior parts of the presomitic mesoderm. These segmented, paired blocks of mesoderm form vertebrae, ribs, skeletal muscles, and subcutaneous tissues. During somitogenesis, the oscillatory expression of Hes7 regulates the periodic segmentation events of the presomitic mesoderm. Hes 7 is a causative gene in the inheritable diseases, spondylocostal dysostoses type 4 and congenital scoliosis, which exhibit developmental bone and vertebral defects (Sparrow, et al., 2008; Sparrow et al., 2012).

\section{Hes functions in the central nervous system}

\subsection{Hes genes in neural stem cells}

Hes genes play crucial roles in inhibiting neuronal differentiation and promoting the proliferation of neural stem (NS) cells in the developing central nervous system (Ohtsuka et al., 2001). During neural development, neuroepithelial cells appear initially and undergo extensive self-renewal. As development proceeds, neuroepithelial cells are gradually elongated and become radial glial cells, which have a cell body in the inner region, called the ventricular zone, and a radial fiber extending from the internal surface to the pial (outer) surface of the brain. Both neuroepithelial cells and radial glial cells are considered to be NS cells. NS cells are known to change their competency over time to generate distinct types of neurons. Radial glial cells divide asymmetrically, forming one radial glial cell and one neuron or basal progenitor from each cell division. Basal progenitors proliferate and produce more neurons in the subventricular zone. After producing neurons, NS cells give rise to oligodendrocytes and astrocytes (Fig. 3A). Hes 1 and Hes 3 are widely expressed by neuroepithelial cells, whereas Hes 1 and Hes5 are mainly expressed by radial glial cells (Hatakeyama et al., 2004).

Hes1, Hes3, and Hes5 are important for maintaining NS cells. Inactivation of these Hes genes leads to failure of maintenance of the undifferentiated state, accelerating neuronal differentiation. As a result, virtually all NS cells become neurons and are therefore depleted prematurely (Hatakeyama et al., 2004). In the absence of these Hes genes, proneural bHLH genes, such as Mash1, Neurogenin2 (Ngn2), and others, are highly up-regulated, leading to premature neuronal differentiation. On the other hand, miss-expression of Hes1 or Hes5 inhibits neuronal differentiation and maintains NS cells (Ohtsuka et al., 2001). Based on their expression patterns, Hes1 and 
Hes 3 are important for the maintenance of neuroepithelial cells, while Hes1 and Hes5 are required for most radial glial cells (Hatakeyama et al., 2004), although these Hes factors are mostly redundant and compensate each other.

\subsection{Hes genes in brain morphogenesis}

Hes genes play important roles in brain morphogenesis (Kageyama et al., 2007). The developing nervous system is partitioned into many compartments by boundary structures, such as the zona limitans intrathalamica, the isthmus, the floor plate and the roof plates (Fig. 3B). The zona limitans intrathalamica is the boundary between the thalamus and the prethalamus; the isthmus is the boundary between the midbrain and the hindbrain; and the floor and roof plates are the boundaries between the right and the left parts of the neural tube. Cells in these boundary structures proliferate poorly and do not give rise to neurons, suggesting that the boundary cells are quiescent, whereas cells in compartments between boundaries proliferate extensively, maintain the NS state, and differentiate into neurons. Several knockout mouse studies showed that the zona limitans intrathalamica is lost in the absence of Hes 1 and Hes5, and that the isthmus and the floor and roof plates are not formed in Hes 1/3/5 triple knockout mice. In the absence of Hes genes, ectopic neuronal differentiation occurs in these defective boundary regions, collapsing the boundary structures (Hirata, et al., 2001). Forced expression of Hes1 in compartment cells inhibits both cell proliferation and neuronal differentiation by repressing cell cycle regulator genes and proneural genes (Baek, et al., 2006; Shimojo, et al., 2008). These results demonstrate that Hes genes are crucial to maintaining these boundary structures in the developing brain by inhibiting differentiation and proliferation of their cells.

Hes1 regulates the maintenance of both quiescent boundary cells and proliferating compartment NS cells, but how does it control two opposite cell cycle states (quiescent versus proliferating)? It has been shown that the expression of Hes 1 is different in these two regions (Baek et al., 2006). Hes1 protein levels are persistent and high in boundary cells, whereas they are variable in individual compartment cells (Fig. 3B). It was recently found that this variability in Hes1 levels in compartments is due to oscillatory expression (see below) (Shimojo et al., 2008). These different expression patterns of Hes1 may be responsible for its different regulatory influences in compartment and boundary cells: repressed proliferation and differentiation in boundary cells, and intense proliferation and neuronal differentiation in compartment cells. 


\section{Hes1 oscillation and cell proliferation in cultured cells}

Hes1 expression oscillates in many types of cultured cells, including fibroblasts, myoblasts, and neuroblasts, and the oscillatory expression can be induced following serum stimulation or Notch activation (Hirata et al., 2002; Masamizu et al., 2006). The period of Hes 1 oscillation is about $2 \mathrm{~h}$ in many mouse cell lines. Hes7, which also oscillates with a 2-h periodicity in the presomitic mesoderm of mouse embryos, acts as a molecular clock to regulate the periodic somite segmentation (Pourquié, 2011). This oscillatory expression is regulated by a negative feedback loop (Fig. 4A). After the activation of Hes 1 transcription by Notch signaling or others, translated Hes1 protein represses its own expression by directly binding to N-box sequences in its promoter. Hes 1 gene products, both mRNA and protein, are very unstable, with a very short half-life of about $20 \mathrm{~min}$ and therefore disappear rapidly, indicating that this auto-repression is short and transient. Disappearance of Hes1 protein allows the next round of expression. In this way, Hes1 autonomously initiates oscillatory expression with a short periodicity. Real-time imaging analyses of Hes1 expression in single cells revealed that the oscillatory expression of Hes1 is intrinsic and surely occurs in normal culture conditions even in the absence of special stimulations such as serum shock, which synchronizes Hes1 induction in cell populations (Masamizu et al., 2006).

It has been reported that the oscillatory expression of Hes 1 is regulated by the Jak-Stat signaling pathway in mouse fibroblast cells (Fig. 4B) (Yoshiura et al., 2007). Jak2 kinase activates Stat3 by phosphorylation, and activated Stat3 induces the expression of downstream genes, including Socs3, an inhibitor of Jak2 kinase. Stat3 phospholylation oscillates in an approximately 2-h cycle, depending on the negative-feedback regulation of Jak2 through Socs3 expression. The blockade of Jak2-Stat3-Socs3 oscillation inhibits Hes1 oscillation and stabilizes Hes1 protein levels by a still unknown mechanism.

Results of several studies suggest that Hes1 oscillation and cell proliferation are correlated with each other. Sustained Hes1 expression leads to G1 phase retardation of the cell cycle (Yoshiura et al., 2007). Hes1 can directly or indirectly repress the transcription of cell cycle-related genes, including E2f1, p21Cipl, p27Kip1, and p57Kip2 (Castella, et al., 2000; Georgia, et al., 2006; Hartman et al., 2004; Murata et al., 2005). Hes 1 oscillation may be important for efficient cell proliferation. Hes1 is identified as one of the factors that protects the quiescent state in fibroblast cells by preventing these cells from undergoing differentiation or senescence (Sang, Coller, \& Roberts, 2008). High and sustained expression of Hes1 protects fibroblasts against 
senescence or differentiation, but the expression of dominant negative-type Hes1 mutants allows quiescent fibroblasts to undergo differentiation. Increased expression of Hes 1 has been reported in malignant tumors, including ovarian carcinomas and breast cancers (Wang et al., 2010; Sang, et al., 2010), but the patterns of its expression in these cells remains unknown. It is important to understand the more detailed relationships between Hes1 expression patterns or levels and cell proliferation or quiescent state.

The molecular mechanism by which cells exhibit different (oscillatory versus sustained) expression modes of Hes1 is still unknown. One candidate is the regulation of microRNA-9 (miR-9) (Bonev, et al., 2012; Tan, et al., 2012). MiR-9 negatively regulates Hes1 mRNA stability by interacting with the 3'-UTR of Hes1 mRNA, enabling oscillatory expression of Hes1. MiR-9 expression is absent in the roof plate and floor plate where Hes1 expression is high and sustained. Over-expression of miR-9 induces cell cycle exit and neuronal differentiation, while miR-9 knockdown inhibits neuronal differentiation of NS cells and dampens Hes1 oscillation.

\section{Hes1 oscillation in embryonic stem (ES) and NS cells}

\subsection{Hes1 oscillation in ES cells.}

ES cells are pluripotent stem cells derived from the inner cell mass of blastocyst-stage embryos. Due to their pluripotency, these cells have the ability to differentiate into various cell types belonging to all three germ layers: ectoderm, mesoderm and endoderm (Smith, 2001). ES cells are known to exhibit heterogeneous gene inductions in response to the same differentiation cues, but the basic mechanism governing such heterogeneity in ES cells is not fully understood. Hes 1 oscillation is one of the candidates for regulating heterogeneous differentiation of ES cells (Kobayashi et al., 2009; Kobayashi \& Kageyama, 2010a). Hes1 is highly expressed in mouse ES cells under the control of leukemia inhibitory factor (LIF) and bone morphogenetic protein (BMP), two essential factors for mouse ES cell culture, and the expression oscillates in individual mouse ES cells with a 3-5-h periodicity, a longer period than that observed in fibroblast cells. This Hes1 oscillation contributes to the heterogeneous properties of ES cell differentiation: Hes1 protein-low cells are prone to differentiate into neuroectodermal cells, while Hes1 protein-high cells differentiate into early mesodermal cells (Fig. 5). Although Hes1 oscillation is dispensable for ES cell pluripotency (Hes 1-null ES cells maintain their pluripotent states in cultured ES cells), Hes 1 oscillation regulates the fate determination step during ES cell differentiation. In 
ES cells, Hes1 regulates Notch signaling molecules and cell cycle-related molecules, both of which are expressed in an oscillating manner in ES cells, suggesting that Hes1 oscillation sets the heterogeneous activation of Notch signaling and the variable timing of cell cycle exit in genetically identical ES cells (Kobayashi \& Kageyama, 2010b). Other fluctuating molecules, including Nanog, Rex1, and Stella, have been identified in ES cells, so they may also contribute more diverse responses in cells to produce various kinds of cells at once even in the same environment (Chambers et al., 2007; Toyooka, et al., 2008; Hayashi, et al., 2008; Kobayashi \& Kageyama, 2011). One attractive hypothesis is that such fluctuating and oscillatory expression of genes is a common feature of stem cells to endow them with their specific properties (Chang, et al., 2008; Furusawa \& Kaneko, 2001, 2012).

\subsection{Hes1 oscillation in developing mouse brain}

Hes 1 expression oscillates dynamically in NS cells both in mouse embryonic brain and in in vitro cultured mouse NS cells with a periodicity of about about 2-3 h (Shimojo et al., 2008). The period and amplitude are variable from cell to cell and from cycle to cycle, suggesting that this oscillation does not serve as an accurate molecular clock but rather enables various (not synchronizing) responses to be individually timed. In NS cells, Hes1 represses the expression of proneural genes, Notch signaling molecules, and cell cycle regulators. Interestingly, time-lapse imaging analyses show that both the proneural gene $\mathrm{Ngn} 2$ and the gene for the Notch ligand Delta-like 1 (Dlll) are expressed in an oscillatory manner in NS cells (Fig. 6A). These oscillations are specific to undifferentiated NS cells and are not observed in differentiating neurons. It was previously thought that Ngn2 and Dll1 expression did not occur in NS cells, but were specific to differentiating neurons. Ngn2 up-regulates the expression of Dll1, which activates Hes1 via Notch signaling in neighboring cells to repress Ngn2 and Dll1 expression and maintain these cells in an undifferentiated state. Thus, Notch signaling is unidirectional, from neurons to NS cells, in this context. However, Ngn2 and Dll1 are actually expressed by NS cells in an oscillatory manner, which is probably dependent on periodic repression by Hes1 oscillation. This oscillatory expression provides another interesting strategy to maintain NS cells in their undifferentiated state. Oscillatory expression of Ngn2 activates only early-response genes such as D111 without activation of the neuronal differentiation program. The oscillatory expressions of Dll1 and Ngn2 maintain proliferating NS cell populations by mutual activation of Notch signaling (Fig. $6 \mathrm{~B}$, left). The expression of Ngn2 becomes sustained when Hes 1 expression is repressed, 
thereby promoting neuronal differentiation (Fig. 6B, right).

\subsection{Hes1 oscillation in NS cells and the optogenetic control}

NS cells are multipotent, generating neurons, olligodendrocytes and astrocytes. NS cells, derived from the ventral telencephalon of mouse embryos, express Hes 1, Mash1, and Olig2. Mash1 promotes cell cycle exit and neuronal fate determination; Olig2 regulates oligodendrocyte specification, and Hes1 regulates the maintenance and proliferation of NS cells. Importantly, these three factors are also reported to function in other events: Mash1 upregulates the gene expression for cell cycle progression (Castro et al., 2011), Olig2 functions in NS cell proliferation (Ligon et al., 2007), and Hes1 promotes astrocyte differentiation (Ohtsuka et al., 2001). Thus, each of these factors has two contradictory functions: maintenance and proliferation of NS cells and determination of a specific cell fate. Time-lapse imaging of these proteins in single NS cells revealed that their gene expressions oscillate: the levels of both Hes 1 and Mash1 proteins oscillate with a periodicity of 2-3 h, and that of Olig2 oscillates with a longer periodicity (5-8 h) (Imayoshi et al., 2013). Moreover, these gene expressions change dynamically when NS cells are differentiating. During neuronal differentiation, Hes1 expression is repressed before asymmetric cell division, when one NS cell produces one neuron and one NS cells. Mash1 protein gradually accumulates and expression becomes sustained in differentiating neurons, whereas Hes1 and Mash1 oscillations resume in daughter NS cells. During astrocyte differentiation, Hes1 protein continues to oscillate but the expression level becomes higher than in NS cells, and then Mash1 and Olig2 expressions become undetectable. During oligodendrocyte differentiation, Olig2 protein expression oscillates at high levels, and Mash1 and Hes1 are down-regulated. These observations reveal that one of the bHLH factors, which are fluctuating in NS cells, becomes dominant and sustained during differentiation (Fig. 6C).

A light-switchable gene expression system is able to generate the oscillatory gene expression with short periodicity. The system makes use of the Neurospora crassa photoreceptor Vivid that is fused with the Gal4 DNA binding domain and the p65 activation domain (GAVPO) (Wang, et al., 2012). Blue light illumination induces the formation of GAVPO homodimers, which bind to UAS sequences and activate transcription of target genes under the control of UAS sequences. A Mash1-inducible system with both Mash1 coding region and its 3'-UTR under the control of UAS sequences in Mash1-null NS cells reveals the importance of gene expression modes (Imayoshi et al., 2013). Repeated exposure to blue light at 3-h intervals successfully 
generates oscillatory expression of Mash1 with a 3-h period, which enhances the cell cycle progression of NS cells. On the other hand, repeated exposure at 30-min intervals, which generates sustained expression of Mash1, increases neuronal differentiation. These results clearly demonstrate that the differences in expression modes of these factors are important for a cell's switch between proliferation and differentiation (Imayoshi et al., 2013). The detailed molecular mechanism by which the oscillatory expression up-regulates the cell cycle progression genes remains unclear.

\section{Conclusions and Perspectives}

Hes factors regulate the maintenance of stem cells and the cell fate decision into multiple cell types in various tissues under multiple signaling pathways, including Notch signaling as described here. These functions are sometimes contradictory to one another. Different expression dynamics is clearly one of the biological strategies to resolve such complex regulations. We mainly focused on Hes1 oscillation in cultured cells, ES cells, and NS cells. It will be interesting to study the role of Hes factor oscillations in other (periodic) developmental processes using imaging techniques. It is also important to address the detailed molecular mechanisms maintaining Hes1 oscillation with biochemical studies and those that switch the expression modes between oscillatory and sustained expressions.

Oscillations were also reported in some signaling pathways regulating Hes factors, such as Notch, Jak-Stat, and Fgf signalings (Kageyama, et al., 2010; Niwa et al., 2007; Yoshiura et al., 2007). For example, Notch and Fgf signaling cooperatively regulate Hes7 oscillation and achieve a spatiotemporal regulation during somite segmentation events (Niwa et al., 2011). Oscillatory Hes7 expression is essential for maintaining periodic somite segmentation. Fgf signaling is important for initiating periodic Hes7 expression in the posterior end of the presomitic mesoderm, and Notch signaling synchronizes and amplifies Hes7 expression from the posterior to the anterior end, thereby inducing periodic somite segmentation at the anterior end. At the same time, Hes 7 connects two signaling pathways by regulating the expression of signaling factors involved in both pathways, leading to coupled oscillation of the two pathways. Such oscillatory coupling between multiple signals via Hes factors may be important for other kinds of tissue development as well.

In mouse embryos, Hes7 expression oscillates with a 2-h cycle, and somite segmentation events also occur with a 2 -h cycle. It is known that the period of somite segmentation is different among different species: $30 \mathrm{~min}$ in zebrafish, $90 \mathrm{~min}$ in chick, 
and $8 \mathrm{~h}$ in human. In the case of Hes1 oscillation in cultured cells, the period is variable between cell types derived from the same species. It is 2-3 hours in NS cells and 3-5 hours in ES cells. It is not known how different cells or cell types set the lengths of these cycles and what the importance of the differential cycle lengths may be. Reconstitution and regulation of the oscillatory expressions using light-inducible systems such as the GAVPO system is an attractive tool not only to reveal the function of oscillation itself but also to understand the significance of the oscillation period.

\section{References}

Akazawa, C., Sasai, Y., Nakanishi, S., \& Kageyama, R. (1992). Molecular characterizationof a rat negative regulator with a basic helix-loop-helix structure predominantly expressed in the developing nervous system. J. Biol. Chem., 267, 21879-21885.

Bae, S.-K., Bessho, Y., Hojo, M., \& Kageyama, R. (2000). The bHLH gene Hes6, an inhibitor of Hes1, promotes neuronal differentiation. Development, 127, 2933-2943.

Baek, J. H., Hatakeyama, J., Sakamoto, S., Ohtsuka, T., \& Kageyama, R. (2006). Persistent and high levels of Hes 1 expression regulate boundary formation in the developing central nervous system. Development, 133, 2467-2476.

Bessho, Y., Miyoshi, G., Sakata, R., \& Kageyama, R. (2001a). Hes7: a bHLH-type repressor gene regulated by Notch and expressed in the presomitic mesoderm. Genes to Cells, 6, 175-185.

Bessho, Y., Sakata, R., Komatsu, S., Shiota, K., Yamada, S., \& Kageyama, R. (2001b). Dynamic expression and essential functions of Hes 7 in somite segmentation. Genes \& Dev., 15, 2642-2647.

Bonev, B., Stanley, P., \& Papalopulu, N. (2012). MicroRNA-9 modulates Hes1 ultradian oscillations by forming a double-negative feedback loop. Cell Rep., 2, $10-18$.

Buscarlet, M., Perin, A., Laing, A., Brickman, J.M., and Stifani, S. (2008). Inhibition of cortical neuron differentiation by Groucho/TLE1 requires interaction with WRPW, but not Eh1, repressor peptides. J. Biol. Chem., 283, 24881-24888.

Castella, P., Sawai, S., Nakao, K., Wagner, J. A., \& Caudy, M. (2000). HES-1 repression of differentiation and proliferation in PC12 Cells: role for the helix 3-helix 4 domain in transcription repression. Mol. Cell. Biol., 20, 6170-6183. 
Castro, D.S., Martynoga, B., Parras, C., Ramesh, V., Pacary, E., Johnston, C., Drechsel, D., Lebel-Potter, M., Garcia, L.G., Hunt, C., Dolle, D., Bithell, A., Ettwiller, L., Buckley, N., \& Guillemot, F. (2011). A novel function of the proneural factor Ascl1 in progenitor proliferation identified by genome-wide characterization of its targets. Genes Dev. 25, 930-945.

Chambers, I., Silva, J., Colby, D., Nichols, J., Nijmeijer, B., Robertson, M., et al. (2007). Nanog safeguards pluripotency and mediates germline development. Nature, 450, 1230-1234.

Chang, H. H., Hemberg, M., Barahona, M., Ingber, D. E., \& Huang, S. (2008). Transcriptome-wide noise controls lineage choice in mammalian progenitor cells. Nature, 453, 544-547.

Espinosa, L., Cathelin, S., D’Altri, T., Trimarchi, T., Statnikov, A., Guiu, J., et al. (2010). The Notch/Hes1 pathway sustains NF-kB activation through CYLD repression in T cell leukemia. Cancer Cell, 18, 268-281.

Fischer, A., \& Gessler, M. (2007). Delta-Notch-and then? Protein interactions and proposed modes of repression by Hes and Hey bHLH factors. Nucleic Acids Res., $35,4583-4596$.

Fukuda, A., Kawaguchi, Y., Furuyama, K., Kodama, S., Horiguchi, M., Kuhara, T., et al. (2006). Ectopic pancreas formation in Hes1 -knockout mice reveals plasticity of endodermal progenitors of the gut, bile duct, and pancreas. J Clin Invest., 116, 1484-1493.

Furusawa, C., \& Kaneko, K. (2001). Theory of robustness of irreversible differentiation in a stem cell system: chaos hypothesis. J. Theor. Biol., 209, 395-416.

Furusawa, C., \& Kaneko, K. (2012). A dynamical-systems view of stem cell biology. Science, 338, 215-217.

Georgia, S., Soliz, R., Li, M., Zhang, P., \& Bhushana, A. (2006). p57 and Hes1 coordinate cell cycle exit with self-renewal of pancreatic progenitors. Dev. Biol., 298, 22-31.

Guiu, J., Shimizu, R., D’Altri, T., Fraser, S. T., Hatakeyama, J., Bresnick, E. H., et al. (2013). Hes repressors are essential regulators of hematopoietic stem cell development downstream of Notch signaling. J. Exp. Med., 210, 71-84.

Hartman, J., Müller, P., Foster, J. S., Wimalasena, J., Gustafsson, J.-A. \& Ström, A. (2004). HES-1 inhibits 17beta-estradiol and heregulin-beta1-mediated upregulation of E2F-1. Oncogene, 23, 8826-8833.

Hatakeyama, J., Bessho, Y., Katoh, K., Ookawara, S., Fujioka, M., Guillemot, F., et al. (2004). Hes genes regulate size, shape and histogenesis of the nervous system by 
control of the timing of neural stem cell differentiation. Development, 131, 5539-5550.

Hayashi, K., Lopes, S. M. C. d. S., Tang, F., \& Surani, M. A. (2008). Dynamic equilibrium and heterogeneity of mouse pluripotent stem cells with distinct functional and epigenetic states. Cell Stem Cell, 3, 391-401.

Hirata, H., Ohtsuka, T., Bessho, Y., \& Kageyama, R. (2000). Generation of structurally and functionally distinct factors from the basic helix-loop-helix gene Hes3 by alternative first exons. J. Biol. Chem., 275, 19083-19089.

Hirata, H., Tomita, K., Bessho, Y., \& Kageyama, R. (2001). Hes1 and Hes3 regulate maintenance of the isthmic organizer and development of the mid/hindbrain. The EMBO J., 20, 4454-4466.

Hirata, H., Yoshiura, S., Ohtsuka, T., Bessho, Y., Harada, T., Yoshikawa, K., et al. (2002). Oscillatory expression of the bHLH factor Hes 1 regulated by a negative feedback loop. Science, 298, 840-843.

Imayoshi, I., Isomura, A., Harima, Y., Kawaguchi, K., Kori, H., Miyachi, H., et al. (2013). Oscillatory control of factors determining multipotency and fate in mouse neural progenitors. Science, 342, 1203-1208.

Iso, T., Sartorelli, V., Chung, G., Shichinohe, T., Kedes, L., \& Hamamori, Y. (2001). HERP, a new primary target of Notch regulated by ligand binding. Mol. Cell. Biol., 21, 6071-6079.

Jensen, J., Pedersen, E. E., Galante, P., Hald, J., Heller, R. S., Ishibashi, M., et al. (2000). Control of endodermal endocrine development by Hes-1. Nature Genetics, 24, 36-44.

Kageyama, R., Hatakeyama, J., \& Ohtsuka, T. (2006). Roles of Hes bHLH fators in neural development. Transcription factors in the nervous system, Wiley-VDH, 3-18.

Kageyama, R., Niwa, Y., Shimojo, H., Kobayashi, T., \& Ohtsuka, T. (2010). Ultradian oscillations in Notch signaling regulate dynamic biological events. Current Topics in Developmental Biology, 92, 311-331.

Kageyama, R., Ohtsuka, T., \& Kobayashi, T. (2007). The Hes gene family: repressors and oscillators that orchestrate embryogenesis. Development, 134, 1243-1251.

Katoh, M., \& Katoh, M. (2007). Integrative genomic analyses on HES/HEY family: Notch-independent HES1, HES3 transcription in undifferentiated ES cells, and Notch-dependent HES1, HES5, HEY1, HEY2, HEYL transcription in fetal tissues, adult tissues, or cancer. International Journal of Oncology, 31, 461-466. 
Kobayashi, T., \& Kageyama, R. (2010a). Hes1 oscillation : making variable choices for stem cell differentiation. Cell Cycle, 9, 207-208.

Kobayashi, T., \& Kageyama, R. (2010b). Hes1 regulates embryonic stem cell differentiation by suppressing Notch signaling. Genes to Cells, 15, 689-698.

Kobayashi, T., \& Kageyama, R. (2011). Hes 1 oscillations contribute to heterogeneous differentiation responses in embryonic stem cells. Genes, 2, 219-228.

Kobayashi, T., Mizuno, H., Imayoshi, I., Furusawa, C., Shirahige, K., \& Kageyama, R. (2009). The cyclic gene Hes 1 contributes to diverse differentiation responses of embryonic stem cells. Genes \& Dev., 23, 1870-1875.

Ligon, K.L., Huillard E, Mehta S, Kesari S, Liu H, Alberta JA, Bachoo RM, Kane M, Louis DN, Depinho RA, Anderson DJ, Stiles CD, Rowitch DH. (2007).

Olig2-regulated lineage-restricted pathway controls replication competence in neural stem cells and malignant glioma. Neuron 53, 503-517.

Masamizu, Y., Ohtsuka, T., Takashima, Y., Nagahara, H., Takenaka, Y., Yoshikawa, K., et al. (2006). Real-time imaging of the somite segmentation clock: Revelation of unstable oscillators in the individual presomitic mesoderm cells. Proc. Natl. Acad. Sci. USA, 103, 1313-1318.

Murata, K., Hattori, M., Hirai, N., Shinozuka, Y., Hirata, H., Kageyama, R., et al. (2005). Hes 1 directly controls cell proliferation through the transcriptional repression of p27Kip1. Mol. Cell. Biol., 25, 4262-4271.

Nakayama, K., Satoh, T., Igari, A., Kageyama, R., \& Nishida, E. (2008). FGF induces oscillations of Hes1 expression and Ras/ERK activation. Curr. Biol., 18, R332-R334.

Niwa, Y., Masamizu, Y., Liu, T., Nakayama, R., Deng, C.-X., \& Kageyama, R. (2007). The Initiation and propagation of Hes7 oscillation are cooperatively regulated by Fgf and Notch signaling in the somite segmentation clock. Dev. Cell, 13, 298-304.

Niwa, Y., Shimojo, H., Isomura, A., González, A., Miyachi, H., \& Kageyama, R. (2011). Different types of oscillations in Notch and Fgf signaling regulate the spatiotemporal periodicity of somitogenesis. Genes \& Dev., 25, 1115-1120.

Ohtsuka, T., Ishibashi, M., Gradwohl, G., Nakanishi, S., Guillemot, F., \& Kageyama, R. (1999). Hes 1 and Hes5 as Notch effectors in mammalian neuronal differentiation. EMBO J, 18, 2196-2207.

Ohtsuka, T., Sakamoto, M., Guillemot, F., \& Kageyama, R. (2001). Roles of the basic helix-loop-helix genes Hes1 and Hes5 in expansion of neural stem cells of the developing brain. J. Biol. Chem., 276, 30467-30474. 
Pourquié, O. (2011). Vertebrate segmentation: from cyclic gene networks to scoliosis. Cell, 145, 650-663.

Sang, L., Coller, H. A., \& Roberts, J. M. (2008). Control of the reversibility of cellular quiescence by the transcriptional repressor Hes1. Science, 321, 1095-1100.

Sang, L., Roberts, J. M., \& Coller, H. A. Hijacking HES1: how tumors co-opt the anti-differentiation strategies of quiescent cells. Trends in Molecular Medicine, $16,17-26$.

Sasai, Y., Kageyama, R., Tagawa, Y., Shigemoto, R., \& Nakanishi, S. (1992). Two mammalian helix-loop-helix factors structurally related to Drosophila hairy and Enhancer of split. Genes \& Dev., 6, 2620-2634.

Shimojo, H., Ohtsuka, T., \& Kageyama, R. (2008). Oscillations in Notch signaling regulate maintenance of neural progenitors. Neuron, 58, 52-64.

Smith, A. G. (2001). Embryo-derived stem cells: of mice and men. Annu. Rev. Cell. Dev. Biol., 17, 435-462.

Sparrow, Duncan B., Chapman, G., Smith, A. J., Mattar, M. Z., Major, J. A., O'Reilly, V. C., et al. (2012). A mechanism for gene-environment interaction in the eiology of congenital scoliosis. Cell, 149, 295-306.

Sparrow, D. B., GuillŽn-Navarro, E., Fatkin, D., \& Dunwoodie, S. L. (2008). Mutation of HAIRY-AND-ENHANCER-OF-SPLIT-7 in humans causes spondylocostal dysostosis. Human Molecular Genetics, 17, 3761-3766.

Sumazaki, R., Shiojiri, N., Isoyama, S., Masu, M., Keino-Masu, K., Osawa, M., et al. (2004). Conversion of biliary system to pancreatic tissue in Hes1-deficient mice. Nature Genetics, 36, 83-87.

Takata, T., \& Ishikawa, F. (2003). Human Sir2-related protein SIRT1 associates with the bHLH repressors HES1 and HEY2 and is involved in HES1- and HEY2-mediated transcriptional repression. Biochem. Biophys. Res. Commun., $301,250-257$.

Tan, S.-L., Ohtsuka, T., González, A., \& Kageyama, R. (2012). MicroRNA9 regulates neural stem cell differentiation by controlling Hes 1 expression dynamics in the developing brain. Genes to Cells, 17, 952-961.

Tateya, T., Imayoshi, I., Tateya, I., Ito, J., \& Kageyama, R. (2011). Cooperative functions of Hes/Hey genes in auditory hair cell and supporting cell development. Dev. Biol., 352, 329-340.

Tomita, K., Hattori, M., Nakamura, E., Nakanishi, S., Minato, N., \& Kageyama, R. (1999). The bHLH gene Hes1 is essential for expansion of early T cell precursors. Genes \& Dev., 13, 1203-1210. 
Toyooka, Y., Shimosato, D., Murakami, K., Takahashi, K., \& Niwa, H. (2008). Identification and characterization of subpopulations in undifferentiated ES cell culture. Development, 135, 909-918.

Ueo, T., Imayoshi, I., Kobayashi, T., Ohtsuka, T., Seno, H., Nakase, H., et al. (2012). The role of Hes genes in intestinal development, homeostasis and tumor formation. Development, 139, 1071-1082.

Wall, D. S., Mears, A. J., McNeill, B., Mazerolle, C., Thurig, S., Wang, Y., et al. (2009). Progenitor cell proliferation in the retina is dependent on Notch-independent Sonic hedgehog/Hes1 activity. J. Cell Biol., 184, 101-112.

Wang, X., Chen, X., \& Yang, Y. (2012). Spatiotemporal control of gene expression by a light-switchable transgene system. Nature Methods, 9, $266 \bigoplus 269$.

Wang, X., Fu, Y., Chen, X., Ye, J., Lü, B., Lü, F. Y. W., et al. (2010). The expressions of bHLH gene HES1 and HES5 in advanced ovarian serous adenocarcinomas and their prognostic significance: a retrospective clinical study. J Cancer Res Clin Oncol, 136, 989-996.

Wendorff, A. A., Koch, U., Wunderlich, F. T., Wirth, S., Dubey, C., Brüning, J. C., et al. (2010). Hes 1 is a critical but context-dependent mediator of canonical Notch signaling in lymphocyte development and transformation. Immunity, 33, 671-684.

Yoshiura, S., Ohtsuka, T., Takenaka, Y., Nagahara, H., Yoshikawa, K., \& Kageyama, R. (2007). Ultradian oscillations of Stat, Smad, and Hes1 expression in response to serum. Proc. Natl. Acad. Sci. USA, 104, 11292-11297.

\section{Figure legends}

Figure 1. Features of Hes genes and their functions. (A) Comparison of protein homology in human Hes factors (Hes1-7, black) and their Drosophila homologues (hairy and E(spl), gray). Phylogenetic tree was generated by ClustalW software from the amino acid sequence alignment. (B) Structure of Hes1 protein. (C) Dual repressor functions of Hes factors: active repression and passive repression. During active repression, Hes factors bind to the N-box or the class $\mathrm{C}$ site in the promoter regions of target genes and repress their transcription. During passive repression, Hes factors form heterodimers with bHLH activators and inhibit their binding to the $\mathrm{E}$ box in the promoter regions of target genes. 
Figure 2. Notch signaling pathway. The Notch receptor is a transmembrane protein activated by the binding of Notch ligands expressed on the surface of neighboring cells (signal sender). Upon ligand binding, the Notch receptor on the signal receiver cell is sequentially cleaved by the ADAM protease and the gamma-secretase, releasing the intracellular domain of Notch (ICD) from the plasma membrane. ICD translocates into the nucleus and forms a complex with the DNA-binding transcription factor RBP-J. Without the ICD, RBP-J represses target gene expression, but is converted to a transcriptional activator when complexed with the ICD and the co-activators (Co-A). Finally, Notch signaling activation induces the expression of target genes such as Hes and Hey.

Figure 3. Hes functions in the central nervous system. (A) Neural stem cells and their differentiation during neural development (upper panel). Neuroepithelial cells initially proliferate by symmetric cell division, and then become radial glial cells, which sequentially generate neurons by asymmetric cell division. Both neuroepithelial cells and radial glial cells are considered to be neural stem (NS) cells. Radial glial cells eventually differentiate into astrocytes, oligodendrocytes, and ependymal cells. Hes factors, expressed in these NS cells, maintain these NS cells and then promote astrocyte differentiation. Proneural bHLH genes, such as Mash1 and Ngn2, are activated in differentiating neurons, leading to the promotion of neurogenesis. (lower panel) Expression pattern of Hes genes in the ventricular zone.

(B) The structure of the developing central nervous system (CNS) and differential expression of Hes 1 gene. The CNS in the mouse at E10.5 (left) is partitioned into two regions, compartments (a) and boundaries (b). Boundary cells function as organizing centers by expressing several signaling ligands, including Shh, Wnt1, or Fgf8. The temporal expression patterns of Hes1 and Mash1 (right panels) are different in compartment and boundary cells. Hes1 expression is variable and oscillating in NS cells in compartments, but is sustained in boundary cells.

Figure 4. Oscillatory expression of Hes1. (A) Hes1 oscillation is regulated by negative feedback: Hes1 protein represses its own expression by directly binding to N-box sequences in its promoter. Both Hes $1 \mathrm{mRNA}$ and protein are unstable and disappear quickly when the expression is repressed. By this mechanism, Hes1 autonomously initiates oscillatory expression with a periodicity of about $2 \mathrm{~h}$. (B) Jak-Stat signaling regulates Hes1 oscillation. Jak2 activity is regulated via negative feedback by Socs3, a downstream gene of Jak-Stat signaling. The levels of phospholylated Stat3 (Stat3-P) 
and Socs3 are oscillating, which regulate Hes 1 oscillation and cell proliferation.

Figure 5. Hes1 oscillation in ES cells. Hes1 expression oscillates in embryonic stem (ES) cells via LIF and BMP stimulation. When differentiation signals activate ES cell differentiation, cells low in Hes 1 protein levels differentiate into neuroectodermal cells (neuronal fate), and those high in Hes1 protein levels tend to differentiate into early mesodermal cells.

Figure 6. Hes1 oscillation in NS cells. (A) The levels of both Hes1 and Ngn2, encoded by a downstream gene of Hesl, oscillate in neural stem (NS) cells. When Hes1 expression is repressed in differentiating neurons, Ngn2 expression becomes sustained. (B) Hes1 oscillation in NS cells induces the oscillatory expression of Ngn2 and Dll1 by periodic repression. Dll1 oscillation mutually activates Notch signaling between neighboring NS cells, which maintain their proliferation without any help from neurons (Left). When Hes1 is repressed, Ngn2 and Dll1 expressions become sustained in differentiating neurons (Right). Notch signaling is unidirectional from differentiating neurons to NS cells. Sustained Dll1 expression might prevent neurons from receiving Notch signaling by the cis-inhibitory function. (C) In NS cells, oscillatory expression of Hes1, Mash1, and Olig2 are important for cell proliferation and stem cell maintenance: oscillatory expression of several fate-determination factors contributes to the multipotent state of NS cells. When differentiation signals arrive, the expression of a single factor becomes sustained or high and determines the cell fate. 
A

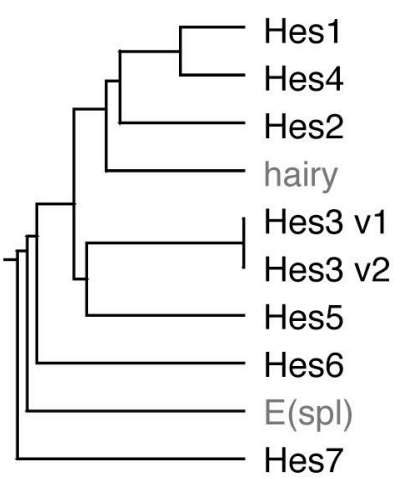

B

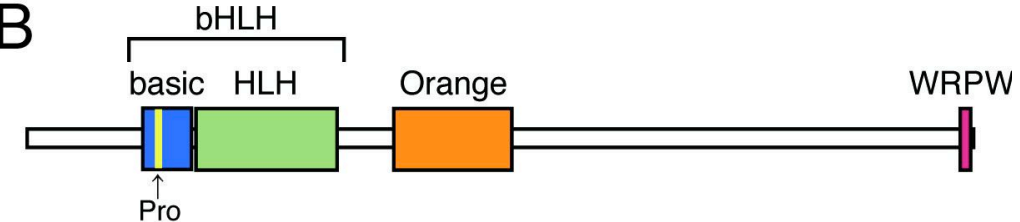

C Active repression

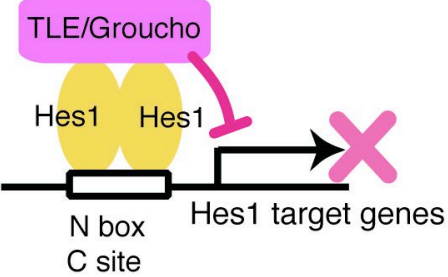

Passive repression

Hes1 E47

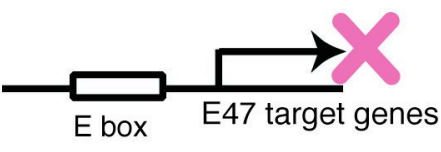

Figure 1 


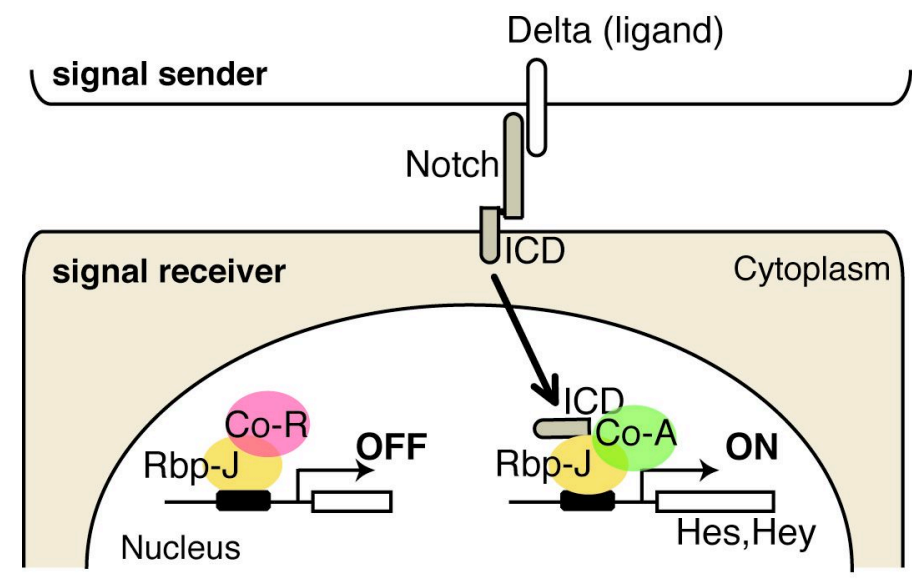

Figure 2 
A

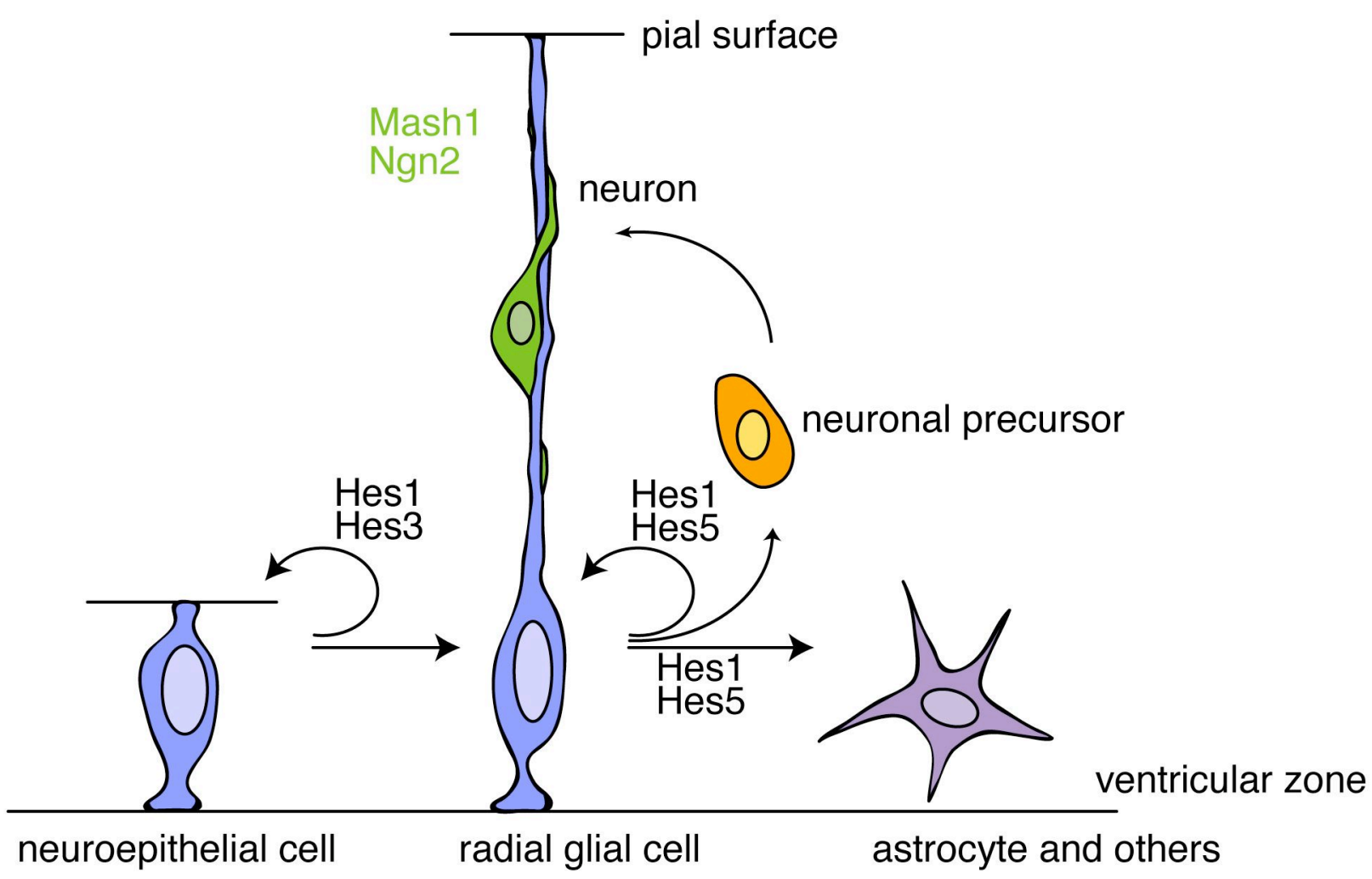

Hes1

Hes3

Hes5

B

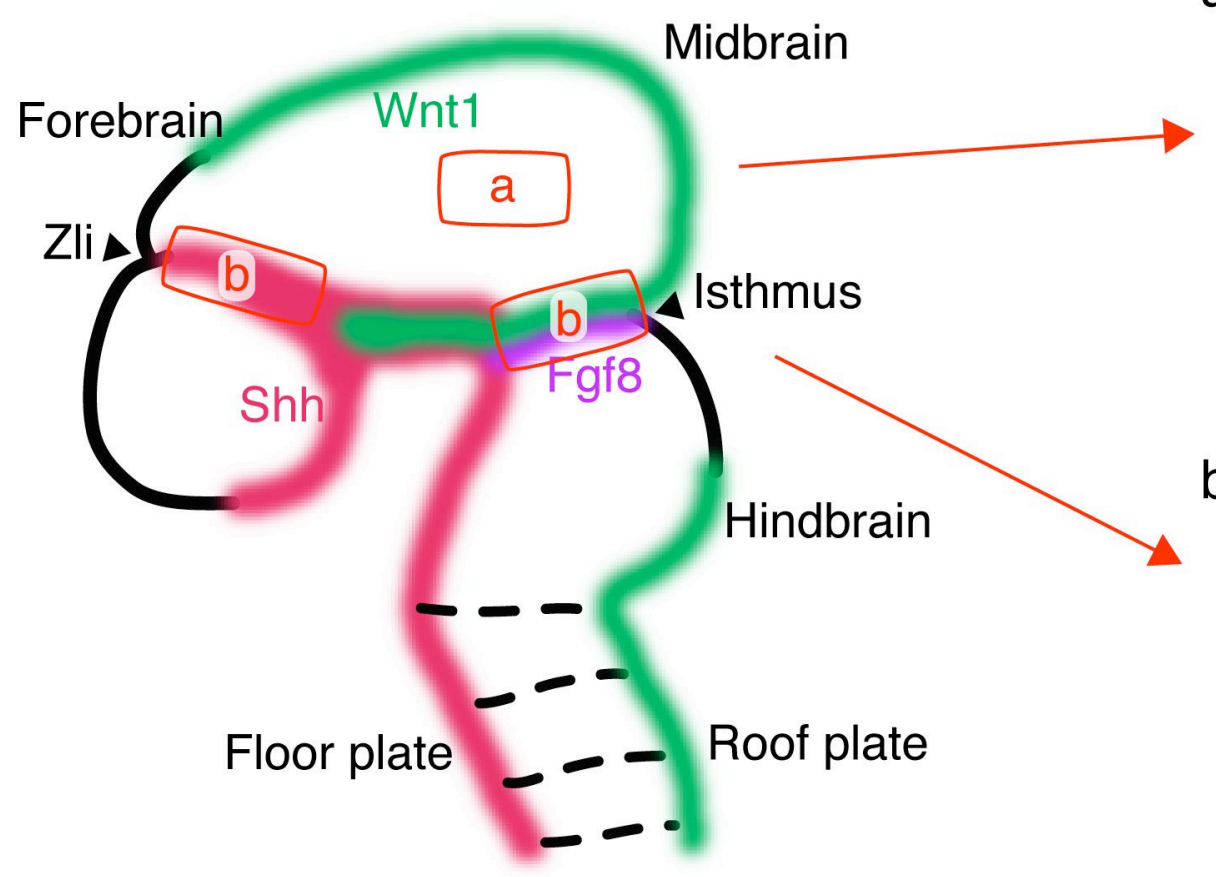

a. compartment

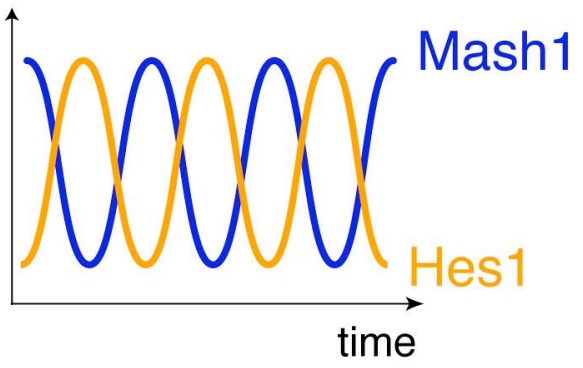

b. boundary

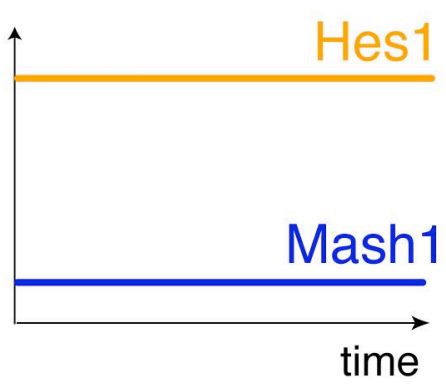

Figure 3 
A

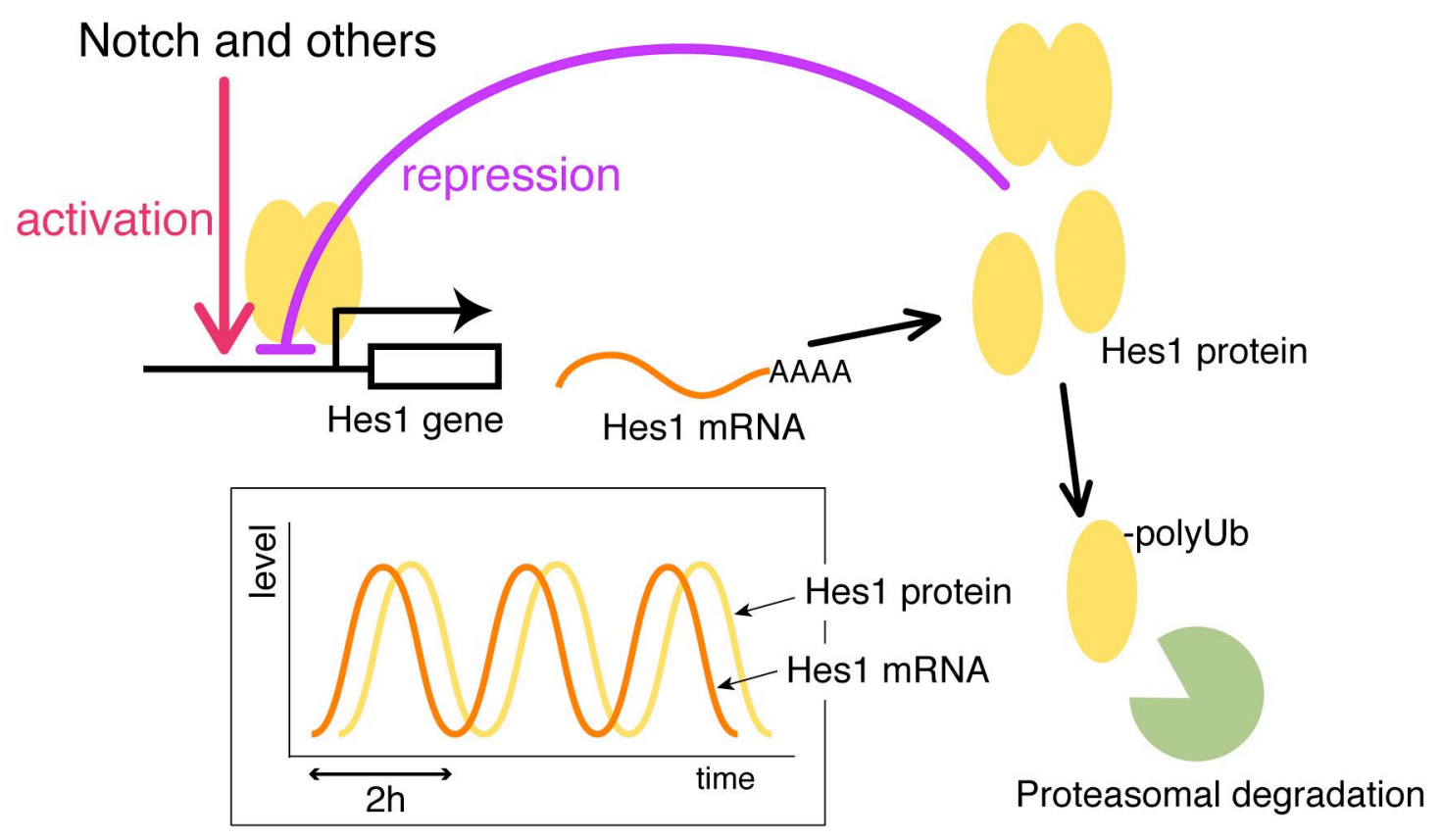

B

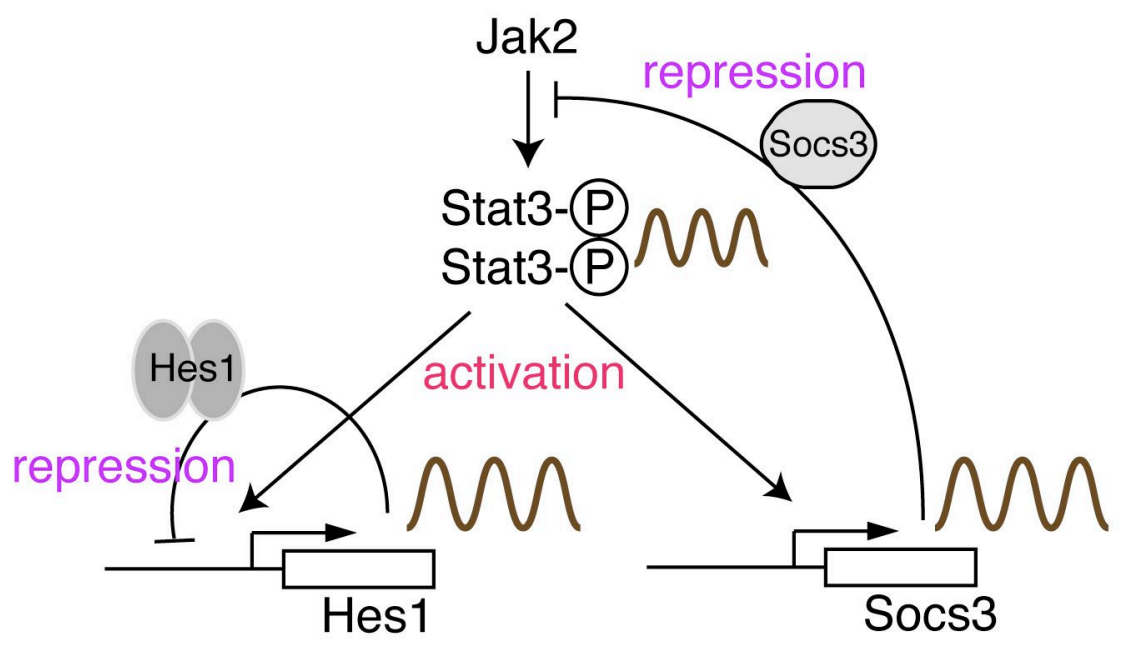

Figure 4 


\section{Differentiation signal}

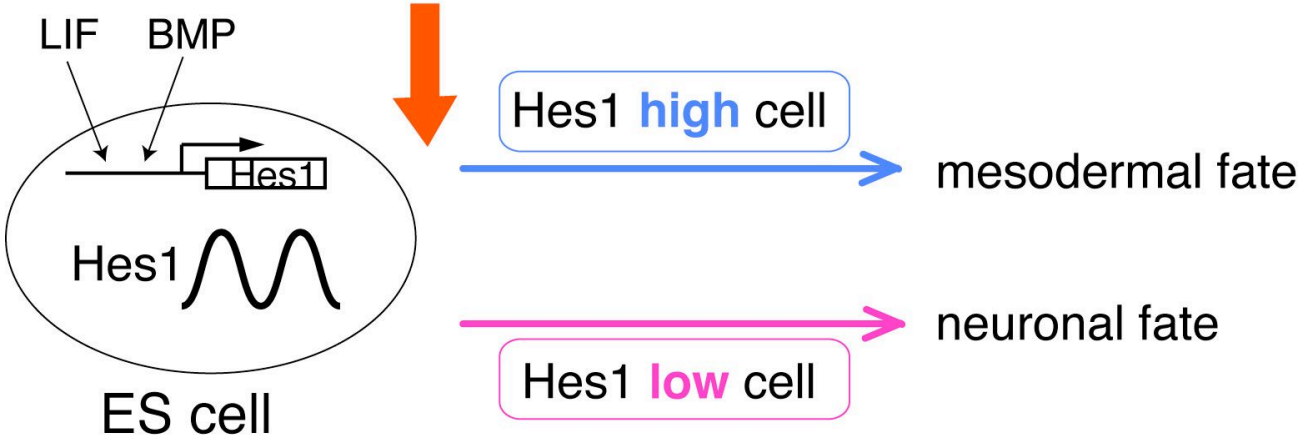

Figure 5 


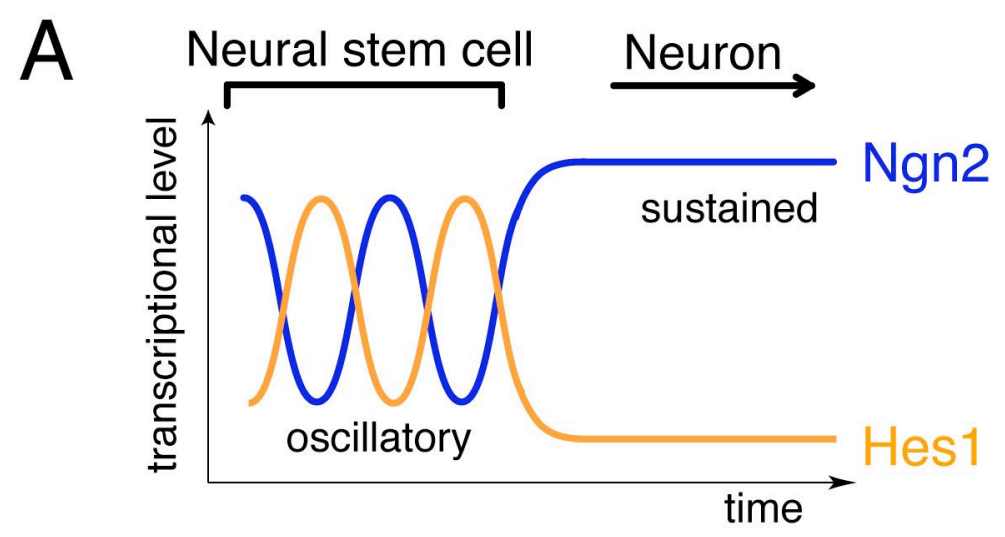

B
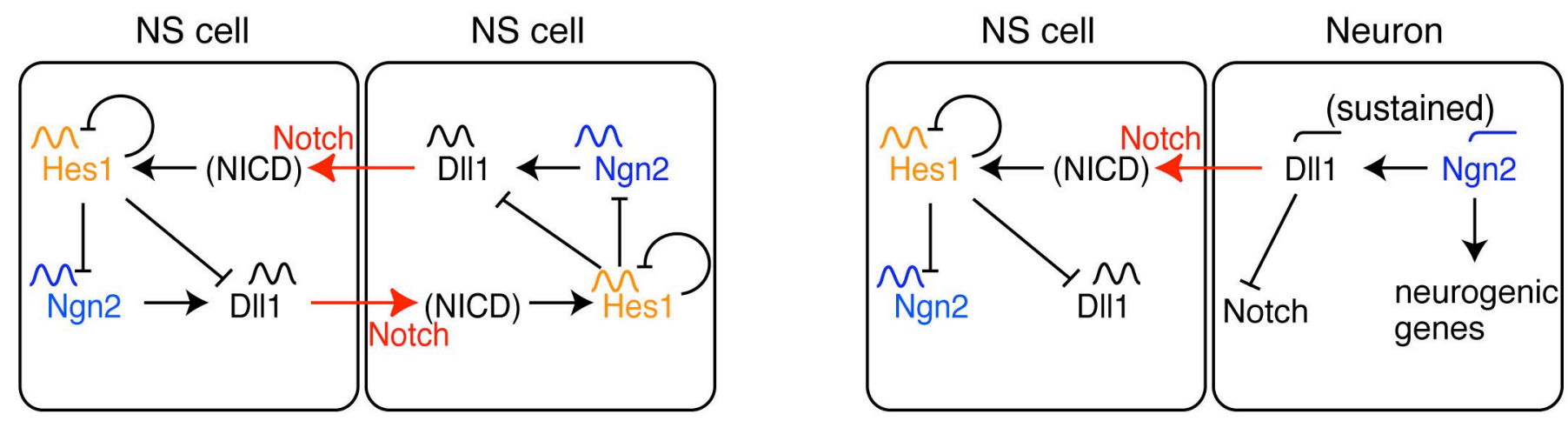

C

\section{Differentiation signal}

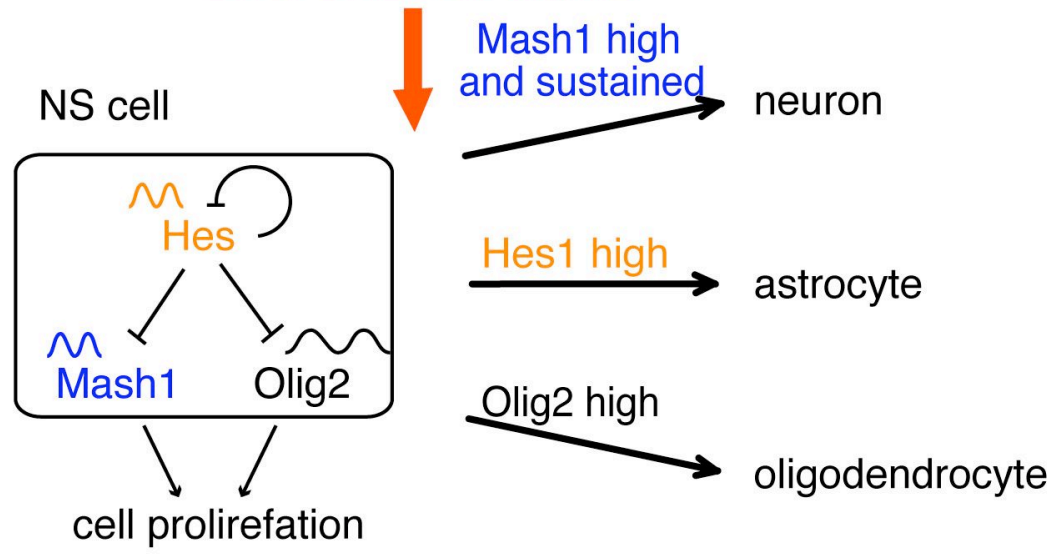

Figure 6 